\title{
Altered TUBB3 expression contributes to the epothilone response of mitotic cells
}

\author{
E Narvi ${ }^{1,2}, K_{\text {Jaakkola }}^{2}$, S Winsel ${ }^{1,2}$, C Oetken-Lindholm ${ }^{2}$, P Halonen ${ }^{2}$, L Kallio ${ }^{2}$ and M J Kallio*,1,2 \\ ${ }^{1}$ Centre of Biotechnology, University of Turku, 20520 Turku, Finland and ${ }^{2}$ Biotechnology for health and well-being, VTT Technical \\ Research Centre of Finland, 20520 Turku, Finland
}

Background: Epothilones are a novel group of microtubule $(\mathrm{mt})$ targeting cancer drugs that bind to the $\beta$-subunit of the $\alpha \beta$-tubulin dimer. Epothilones inhibit cell proliferation and induce cell death by interfering with the normal mt function. In this study, we examined the consequences of altered expression of human $\beta$-tubulin isotypes in terms of the epothilone drug response in human lung and breast cancer cell lines.

Methods: The $\beta$-tubulin isotypes TUBB2A-C, TUBB3 and TUBB were silenced or overexpressed in A549, A549EpoB40 and MCF7 cell lines in the presence or absence of epothilones. The drug effects on cell proliferation, mitosis and mt dynamics were determined using live cell microscopy and immunofluorescence assays.

Results: Loss of TUBB3 enhanced the action of epothilones. TUBB3 knockdown increased the severity of drug-induced mitotic defects and resulted in stabilisation of the mt dynamics in cells. Moreover, exogenous expression of TUBB3 in the epothilone resistant cell line conferred the response to drug treatments. In contrast, reduced levels of TUBB2A-C or TUBB had not apparent effect on the cells' response to epothilones.

Conclusion: Our results show that the expression of TUBB3 contributes to the cellular response to epothilones, putatively by having an impact on the mt dynamics.

Microtubule (mt) targeting drugs such as the taxanes (paclitaxel and docetaxel) and vinca alkaloids (vinblastine and vincristine) are widely recognised as one of the most important chemotherapeutics in the treatment of different types of cancer (Jordan and Wilson, 2004). The family of $\mathrm{mt}$ drugs grows further as a new pharmacophore, the epothilones, is being transferred to clinical practise: ixabepilone (Ixempra; Bristol-Myers Squibb, New York City, NY, USA) is already available for the treatment of breast cancer while sagopilone (Bayer HealthCare, Leverkusen, Germany) and epothilone B (epoB, patupilone, Novartis, Basel, Switzerland) have advanced to clinical trials. Epothilones, taxanes and vinca alkaloids function by disturbing the normal dynamics of the mitotic spindle apparatus. These drugs all bind to the $\beta$-subunit in the $\alpha \beta$-tubulin dimer, but the exact binding site on the filamentous $\mathrm{mts}$ varies between the individual drugs (Downing, 2000). Even though the phenotype of the drug effect, defective mitosis leading to the suppression of cell proliferation, is well documented, the precise events activating the molecular pathways leading to cell death have remained elusive.
The efficiency of the mt targeting chemotherapy is frequently compromised by intrinsic or acquired drug resistance. First, paclitaxel and docetaxel are substrates for the P-glycoprotein (Pgp)-mediated drug efflux pump, which hampers the optimal drug response in patients who overexpress the Pgp (Alvarez et al, 1995). Compared with the taxanes, the epothilones are much less susceptible to efflux pumps (Wartmann and Altmann, 2002) and thus are expected to assist in overcoming the Pgp-mediated drug resistance. Second, the expression of various $\beta$-tubulin isotypes differs considerably among tissues and cell types (Kilpinen et al, 2008). The patient's individual $\beta$-tubulin isotype expression profile has been shown to play an important role in determination of the paclitaxel response. For example, breast and ovarian cancer patients with high TUBB3 (class III $\beta$-tubulin, NM_006086) expressions have increased paclitaxel resistance (Tommasi et al, 2007). Moreover, patients with lung cancer having low amounts of TUBB3 are more sensitive to paclitaxel treatments (Seve et al, 2005). The same phenomenon has also been observed in several 
studies with cancer cell lines; high expression of TUBB3 has been found to be associated with paclitaxel resistance in human lung (Burkhart et al, 2001), ovarian (Kavallaris et al, 1997), prostate (Ranganathan et al, 1998) and breast (Stengel et al, 2010) cancer cells. In addition to frequently reported neuronal expression, TUBB3 is expressed in various normal tissues as well as in important groups of malignancies (Kilpinen et al, 2008).

TUBB (class I $\beta$-tubulin, also called TUBB5, NM_178014) is the most commonly and constitutively expressed $\beta$-tubulin isotype (Kilpinen et al, 2008). Mutations in the TUBB gene can interfere with drug binding and cause resistance to $\mathrm{mt}$ stabilizing drugs, including epothilones (Giannakakou et al, 1997; He et al, 2001; Berrieman et al, 2004; Wiesen et al, 2007; Mozzetti et al, 2008). TUBB2 subfamily consists of three distinct genes; TUBB2A (class IIa $\beta$-tubulin, NM_001069), -2B (class II $\beta$-tubulin, NM_178012) and -2C (class IVb $\beta$-tubulin, NM_006088). TUBB2A and -2B are structurally similar except of one amino acid difference.

To characterise the roles of $\beta$-tubulin isotypes TUBB2A-C, TUBB3 and TUBB in the cellular response to epothilones, we performed silencing and overexpression studies in human lung A549 and breast MCF7 cancer cells. The cell lines were chosen to represent the cancer types against which the epothilones are used in the clinics. We show that the RNAi-mediated silencing of TUBB3-sensitised cells to sagopilone, epothilone B and ixabepilone treatments. The TUBB3 deficient drug-treated cells exhibited significantly more multipolar mitotic spindles and showed decreased cell growth $\left(\mathrm{EC}_{50}\right)$ compared with the drug-treated cells with intact TUBB3 expression. Furthermore, loss of TUBB3 in non-drug-treated cells resulted in stabilisation of the mt dynamics. To investigate the role of TUBB3 in the drug response we utilised A549EpoB40 cell line which is an epothilone/taxane resistant variant of A549 that carries a mutation at $\beta 292$ from Gln to Glu (He et al, 2001). TUBB3 was able to mediate drug response in the epothilone resistant cell line. These results support the notion that determination of the $\beta$-tubulin isotype expression profiles of tumours can possess diagnostic value and predict cancer cell response to epothilones.

\section{MATERIALS AND METHODS}

Cells and drugs. A549 non-small cell lung cancer cells and MCF7 breast cancer cells were from ATCC (Manasses, VA, USA). The drug resistant A549 variant A549EpoB40 was a kind gift from Susan Horwitz (Yang et al, 2005). The anti-mt drugs paclitaxel (Sigma, Poole, UK), epothilone B (Patupilone, Tocris Bioscience, Bristol, UK), ixabepilone (Bristol-Myers Squibb) and sagopilone (Bayer HealthCare AG) were dissolved to cell culture medium to obtain the desired drug concentration.

Antibodies and primers. The validated antibodies used in the study were tubulin isotype specific monoclonal antibodies TUJ-1 (Covance, Princeton, NJ, USA MMS-435 P) for TUBB3, 7B9 (Abcam, Cambridge, MA, USA, ab28035) for TUBB2, SAP.4G5 (Sigma, T7816) for TUBB, YL1/2 (Abcam, ab6160) for TUBA, a mouse anti-GAPDH (Advanced ImmunoChemical Inc., Long Beach, CA, USA) and the rabbit polyclonal anti-pericentrin antibody (Abcam, ab4448). Primers used in Taqman quantitative real-time PCR were for TUBB3 GCAACTACGTGGGCGACT and CGAGGCACGTACTTGTGAGA used together with Universal probe library (Roche, Basel, Switzerland) probe \#78, for TUBB ctgccacatcagtgtttgagtc and aaaaagatggaggagggttcc together with probe \#82, for TUBB2A gacgaacaaggggagttcg and ggatgcacgattgatctgag with probe $\# 63$, for TUBB2B aggacggacagacccagac and ctgatgacctcccaaaacttg with probe \#79, for TUBB2C ctgctgctgttt gtctacttcc and getgatcacctcccaaaact with probe \#38.
Gene silencing experiments. The siRNA oligos used in the study were Thermo scientific (Walthamn, MA, USA) L-010325-00-0005 for TUBB, Qiagen (Hilden, Germany) 1846768, Dharmacon (Walthamn, MA, USA) L-020099-00-0005 and Ambion (Carlsbad, CA, USA) s20296 for TUBB3, Dharmacon L-008260-00-0005 for TUBB2A, Dharmacon L-017790-01-0005 for TUBB2B and Dharmacon L-008265-00-0005 for TUBB2C. 40000 cells (A549 and A549EpoB40) were plated per well in a 24-well plate. The next day, transient transfection was performed using SiLentFect (Bio-Rad, Hercules, CA, USA) transfection reagent according to the manufacturer's instructions using $20 \mathrm{nM}$ of siRNA. For MCF7 cells, transient reverse transfection protocol with SiLentFect reagent, $30 \mathrm{nM}$ siRNA and 60000 cells per well was used according to the manufacturer's instructions. Drug treatments $(20 \mathrm{~h})$ of the cells were started $48 \mathrm{~h}$ after the transfection.

TUBB isotype constructs and transfection. Overexpression of TUBB3 and TUBB was induced by transfecting C-terminal Myc-tag containing expression vectors (Origene, Rockville, MD, USA RC200755 and RC203629, respectively) to the A549 or A549EpoB40 cells. A549 cells stably expressing $\alpha$-tubulin-eGFP (BD Biosciences Clontech, Franklin Lakes, NJ, USA \#632349) or H2B-GFP (Kanda et al, 1998) were created. Transfections were performed using Lipofectamin 2000 (Invitrogen, Carlsbad, CA, USA) transfection reagents according to the manufacturer's instructions. Stable cell lines were created by selecting with G418 (Sigma). The drug treatments and subsequent sample preparation for immunofluorescence were performed as described above.

Western blotting. Cells were harvested by trypsinisation, washed in phosphate-buffered saline (PBS) ( $\mathrm{pH} 7.2)$. Cell pellets were diluted in sample buffer ( $5 \mathrm{ml} \mathrm{I} \mathrm{M} \mathrm{Tris-} \mathrm{HCl} \mathrm{pH} \mathrm{6.8,} 8 \mathrm{ml} 20 \%$ SDS, $2 \mathrm{ml}$ glycerol, $0.308 \mathrm{~g}$ DTT, $0.002 \mathrm{~g}$ bromophenol blue) and heated $90^{\circ} 10 \mathrm{~min}$. Proteins were separated by SDS-PAGE with $4-20 \%$ gradient gel (Bio-Rad) and transferred onto nitrocellulose membrane. Membranes were blocked in $10 \mathrm{~mm}$ Tris- $\mathrm{HCl}(\mathrm{pH}$ 8.0), $150 \mathrm{~mm} \mathrm{NaCl}$ (TBS) containing 5\% non-fat dry milk. The membranes were incubated with antibodies. After washing three times with TBS supplemented with $0.1 \%$ Tween, the membranes were incubated in IRDye Conjugated 680 Goat Anti-Mouse ab. The Odyssey Infrared Imaging System (LI-COR Biotechnology, Lincoln, NE, USA) was used for the detection of proteins.

Immunofluorescence. The cell specimens were fixed with $4 \%$ paraformaldehyde containing $0.05 \%$ glutaraldehyde for $7 \mathrm{~min}$ and blocked with $1 \%$ BSA containing $0.5 \%$ Triton X-100. The fixed cells were stained with primary antibodies (1:500 in PBS) and secondary antibodies (1:500 in PBS). DAPI staining was used to visualise DNA. Coverslips were mounted on Vectashield mounting medium (Vector labs, Burlingame, CA, USA).

Microscopy and image analysis. The stained specimens were analysed using Zeiss (Oberkochen, Germany) Axiovert $200 \mathrm{M}$ microscope equipped with Yokogawa (Tokyo, Japan) CSU22 confocal scanner unit and Intelligent Imaging Innovations (Intelligent Imaging Innovations, Inc., Denver, CO, USA) laser unit microscope using $63 \times$ oil objective. The confocal microscope software (SlideBook 4.2, Intelligent Imaging Innovations, Inc.) was used to analyse the data. In the analysis of mitotic spindle morphology a minimum of 50 mitotic cells were analysed in each specimen and the mitotic cells were classified to bipolar or multipolar cells according to the spindle morphology. Condensed chromosomes were considered as a specification of a mitotic cell. Any mitotic cell having more than two $\mathrm{mt}$ nucleating foci was considered multipolar despite of the mitotic phase of the cell. The silencing efficacy was determined using image based analysis as follows. Silenced and non-silenced cell populations were labelled with isotype specific anti-tubulin antibodies. Through cell image stacks were recorded with $0.5 \mu \mathrm{m}$ step size. Integrated signal 
intensity of anti-tubulin antibody labelled individual cells was measured from 25 cells in silenced and control cell populations in three independent assays.

Determination of $\mathbf{m t}$ dynamics. The $\mathrm{mt}$ dynamics was measured in live A549 cells stably expressing $\alpha$-tubulin-eGFP using a spinning disk confocal microscope with $100 \times$ oil objective. Stacks of eight focus levels in $0.26 \mu \mathrm{m}$ step size were recorded every $10 \mathrm{~s}$ during a filming session of $250 \mathrm{~s}$. The plus ends of mts were digitally marked in the recorded time-lapse movies to allow measurement of the mt dynamicity. Dynamicity refers here to the growth and shrinkage velocities of the mts, which were determined as a measurement of the distance that the $\mathrm{mt}$ plus-end travelled in a specific period of time. Approximately $10 \mathrm{mts}$ per cell and a minimum of five silenced cells per sample were analysed. The plate position coordinates of each TUBB3 silenced cell used in the live cell analysis of mt dynamics was recorded which allowed identification of the same cells after fixation and anti-tubulin immunofluorescence to confirm the TUBB3 silencing at single cell level. Only those cells in which TUBB3 signal intensity after immunofluorescence was below the level of visual detection were included to the analysis.

Measuring cell proliferation. $\mathrm{EC}_{50}$ values were determined as follows. Cells were transfected with TUBB3, TUBB or negative control siRNAs and plated on 384 -well plates $24 \mathrm{~h}$ after transfection. The drugs were added on the cells as concentration series (0.033-100 nM) and the samples were incubated for $48 \mathrm{~h}$. Cell Titer Blue reagent (Promega, Fitchburg, WI, USA) was added to the samples and the fluorescence at 560/590 nm was measured in each well. Corresponding $\mathrm{EC}_{50}$ values for each drug and/or gene silencing combination were determined based on the fluorescence measurements.

Statistical analysis. Statistical analysis was performed with Student's $t$-test using Microsoft Excel or Graph Pad Prism 4 (GraphPad Software, Inc, La Jolla, CA, USA). Analysis of mt dynamics was made using SlideBook 4.2 software and the $\mathrm{EC}_{50}$ value determination with Graph Pad Prism 4. All errors shown are the mean \pm s.e.m.

\section{RESULTS}

Altered expression of individual $\beta$-tubulin isotypes does not induce morphological changes in otherwise non-perturbed cells. To clarify the role of $\beta$-tubulin isotypes in mitosis, we studied how changes in their expression affect cell morphology and mitotic progression. For that purpose, we suppressed the expression of TUBB2A, TUBB2B, TUBB2C, TUBB3 and TUBB in A549 and MCF7 cells using one-to-three individual siRNAs per each tubulin isotype. To analyse the silencing efficacy, we first performed qPCR that indicated significant downregulation of the target gene mRNA at $48 \mathrm{~h}$ after the introduction of the $\beta$-tubulin isotype specific siRNAs into the cells (Figure 1A). Depletion of one $\beta$-tubulin isotype induced some variation in the expression of the other $\beta$-tubulin isotypes (Figure 1A). Next, we determined the silencing efficacy at the protein level $48 \mathrm{~h}$ post-transfection using immunofluorescence with monoclonal anti-TUBB2A/B, anti-TUBB3 and anti-TUBB antibodies (Figure 1B). The analysis indicated significant downregulation of protein levels of all tubulin isotypes targeted with RNAi (reduction by $92-97 \%$ ) based on the quantification of the signal intensities after immunofluorescence. In addition, the depletion of the TUBB and TUBB3 was confirmed at the cell population level using western blotting (Figure 1C). Despite the significant downregulation of the individual $\beta$-tubulin isotypes we did not observe any notable morphological changes in the interphase or mitotic cells; the mt networks of interphase cells were intact and the architecture of spindle apparatus in the $\mathrm{M}$-phase cells was indistinguishable from the controls.
To further analyse the effects of altered $\beta$-tubulin isotype expression on cell morphology, we made overexpression studies with Myc-tagged TUBB3 and TUBB isotypes in A549 cells. AntiMyc antibody staining showed that the Myc-tagged tubulins were incorporated into mt filaments in both interphase and mitotic cells (Supplementary Figure S1). At the population level, the increase in the TUBB or TUBB3 expression was modest (Figure 1C). Importantly, only the bright myc-positive cells were used in the analysis of drug responses. The morphology of the mitotic spindles in the TUBB3 or TUBB overexpressing cells was comparable with the spindles in cells expressing the negative control vector (Supplementary Figure S1).

Loss of TUBB3 leads to stabilisation of mts. In parallel with the analysis of cellular morphology, we studied the effect of TUBB3 silencing on the mt dynamics using live cell imaging of A549 cells stably expressing GFP- $\alpha$-tubulin. TUBB3 siRNA or control siRNA was introduced into the cells and the dynamicity (total growth or shrinkage of $\mathrm{mt}$ plus ends) of individual $\mathrm{mts}$ was determined at 10 second intervals in groups of live cells in both cell populations $48 \mathrm{~h}$ post-transfection (Figure 2A and B). The location of the analysed live cells on the cover slips were recorded that allowed confirmation of the TUBB3 silencing level in the same cells after fixation and immunofluorescence with an anti-TUBB3 antibody. TUBB3 silencing resulted in reduction in the $\mathrm{mt}$ growth/shrinkage rates despite no cell cycle or cell morphology defects were observed in the TUBB3-depleted cells (Figure 2B and C). The mean $\mathrm{mt}$ growth/shrinkage rate of TUBB3 silenced cells was $0.052 \pm 0.009 \mu \mathrm{m} \mathrm{s}^{-1}$, which is significantly different from the $0.080 \pm 0.008 \mu \mathrm{m} \mathrm{s}^{-1}$ of control cells $(P=0.0124)$. We conclude that loss of TUBB3 leads to increased $\mathrm{mt}$ stabilisation in cells.

The effects of epothilones on the morphology of mitotic spindle and $\mathbf{m t}$ dynamics. To determine the effects of epothilones on the mitotic spindle apparatus, we treated A549 and MCF7 cells with sagopilone, epothilone B, ixabepilone or drug-free medium for $20 \mathrm{~h}$ before fixation and immunofluorescence (Figure 3A-E). The cells were stained with anti-pericentrin and anti- $\alpha$-tubulin antibodies to visualise the location and count the number of $\mathrm{mt}$ nucleating centres in groups of at least 50 mitotic cells per each sample. Treatment of cells with $1.0 \mathrm{~nm}$ sagopilone, epothilone B or $3.0 \mathrm{~nm}$ ixabepilone resulted in the formation of abnormal multipolar mitotic spindles and multinuclear interphase cell morphology (Figure $3 \mathrm{~A}$ and $\mathrm{B}$ ). Even subnanomolar concentrations of sagopilone were able to induce multipolarity. However, in most of the mitotic cells having multiple mt nucleating foci only two pericentrin positive centres were observed (Figure 3A). Some variation in the sensitivity of the cell lines to the drugs was observed. MCF7 cells exhibited more multipolar spindles after treatment with 0.5 or $1.0 \mathrm{nM}$ concentrations of sagopilone in comparison to the A549 cells (Figure 3B and E).

The mitotic phenotype was further studied in live cells using GFP-H2B expressing A549 cells treated with various concentrations of sagopilone. As expected, continued presence of $10 \mathrm{nM}$ sagopilone in the culture medium for $20 \mathrm{~h}$ caused accumulation of cells to $M$ phase (mitotic index $90.6 \% \pm 1.4 \%$ ). In contrast, the multipolar cells induced by lower concentrations of sagopilone $(1.0 \mathrm{nM})$ did not cause $\mathrm{M}$ phase delay or arrest (mitotic index $2.8 \% \pm 0.3 \%$ compared with control $2.7 \% \pm 0.2 \%$ ) but rather the cells entered into anaphase, decondensed chromosomes and exited mitosis (Supplementary Figure S2). In these cells, the cytokinesis occasionally failed leading to formation of polyploid multinuclear progeny cells. The polyploid multinuclear phenotype was also frequently observed after treatment of A549 GFP-H2B cells with low concentrations of epothilone B and ixabepilone (data not shown).

Next, we verified the mt stabilising effects of epothilones in live A549 GFP- $\alpha$-tubulin cells by measurements of $\mathrm{mt}$ dynamics. 

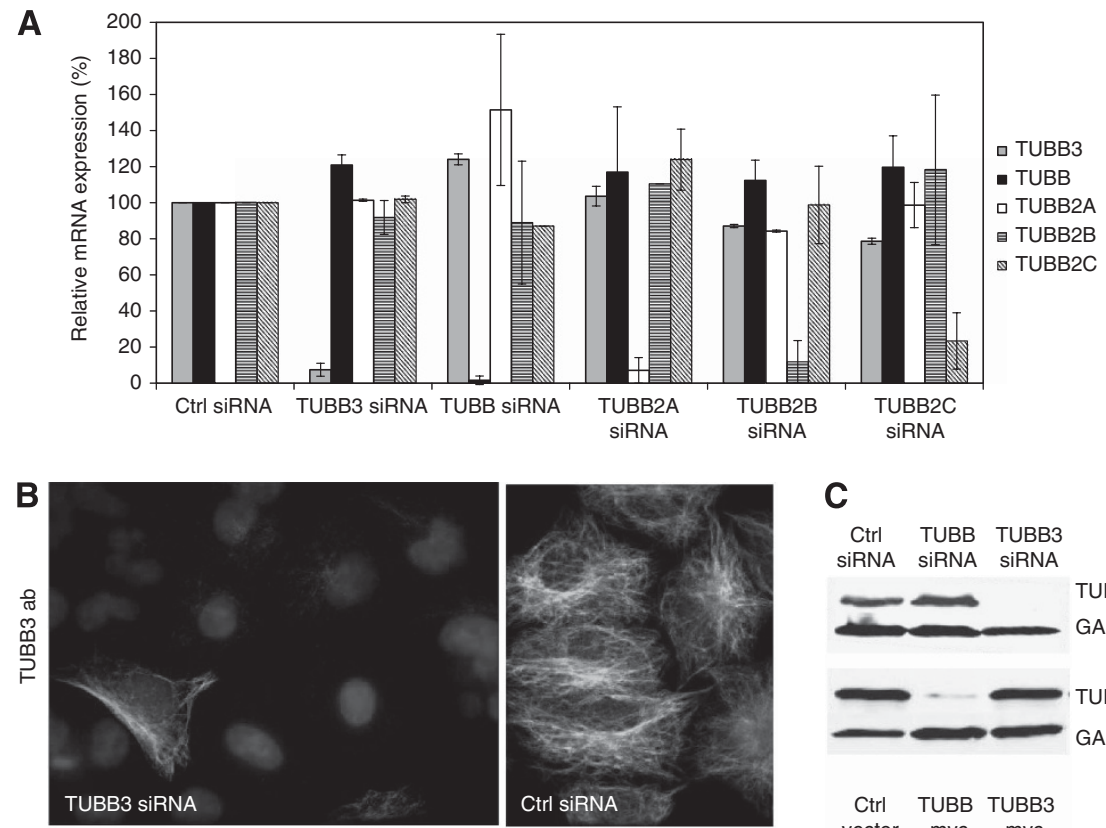

C
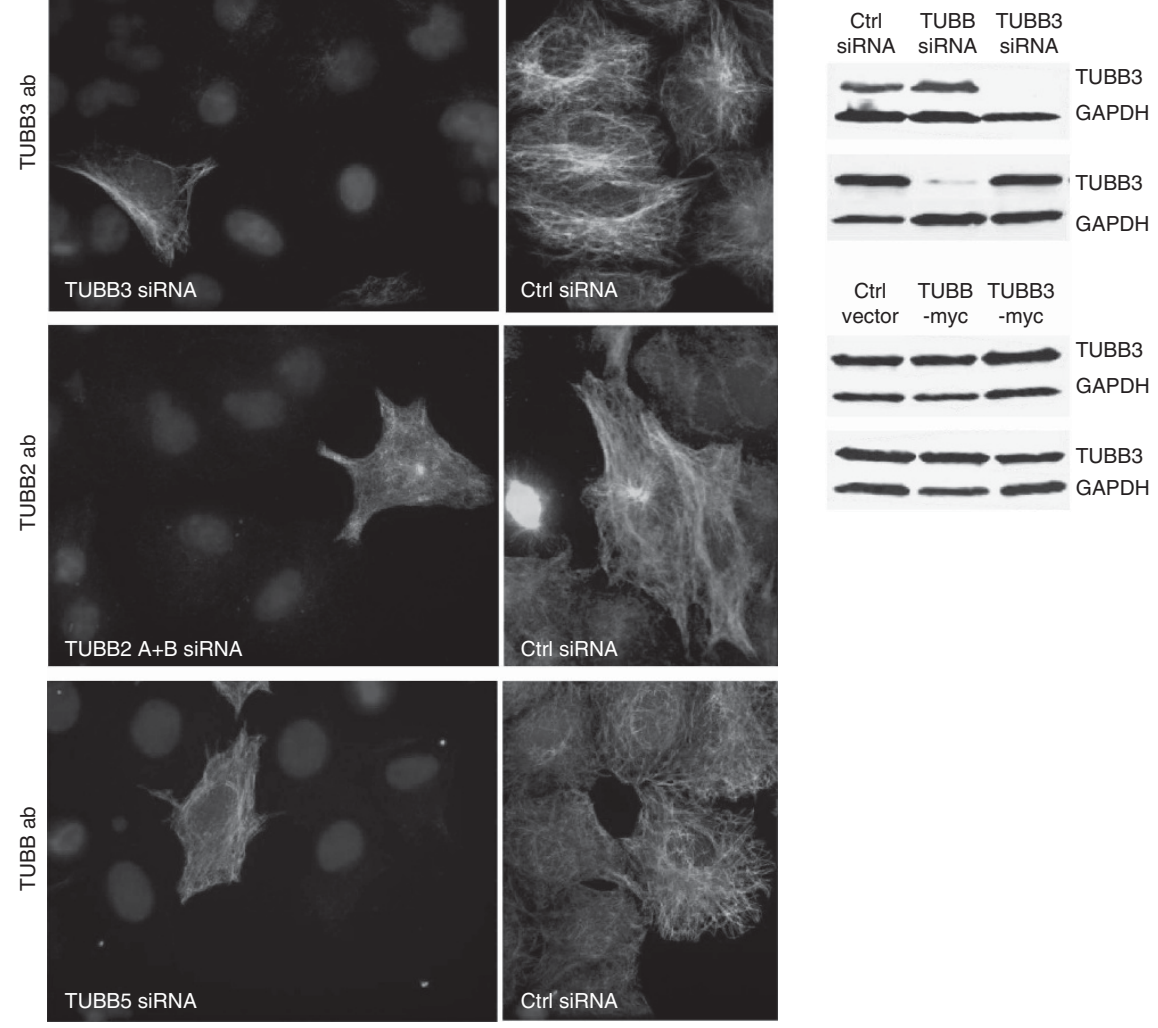

Figure 1. Silencing efficacy of various $\beta$-tubulin isotypes in human A549 lung adenocarcinoma epithelial cell line. (A) Relative mRNA expression of indicated $\beta$-tubulin isotypes in $\beta$-tubulin silenced A549 cells measured by qPCR. Expression of control (Ctrl) siRNA transfected cells is set as $100 \%$ for each isotype. (B) Detection of $\beta$-tubulin isotype specific immunostainings in A549 cells after gene silencing. Cells silenced with TUBB3, TUBB2 or TUBB siRNAs were stained using anti-TUBB3, -TUBB2 or TUBB antibodies, respectively (green). In all micrographs, one non-silenced cell is included to the field of view as a staining control. DNA is stained with DAPI (blue). The scale bar equals $10 \mu \mathrm{m}$. (C) Representative western blots showing the silencing and overexpression of TUBB and TUBB3 in A549 cells.

Treatment of cells with $2.0 \mathrm{~nm}$ sagopilone resulted in a complete loss of mt dynamics (Figure 2B). In further studies, we used $1.0 \mathrm{~nm}$ drug concentration that still was sufficient to stabilise the mts (Figure 2C). Treatment with $1.0 \mathrm{~nm}$ sagopilone significantly reduced the average $\mathrm{mt}$ growth/shrinkage speed to $0.056 \pm 0.009 \mu \mathrm{m} \mathrm{s}^{-1}$ compared with control $0.080 \pm 0.008 \mu \mathrm{m} \mathrm{s}^{-1}$ $(P=0.0256)$. Sagopilone treatment $(1.0 \mathrm{nM})$ did not further stabilise the mts in the TUBB3 silenced cells; the mt growth/ shrinkage speed of sagopilone treated TUBB3 silenced cells was $0.047 \pm 0.007 \mu \mathrm{m} \mathrm{s}^{-1}$ compared $0.050 \pm 0.007 \mu \mathrm{ms}^{-1}$ of nondrug-treated TUBB3 silenced cells (Figure 2C).

$\boldsymbol{\beta}$-Tubulin expression profile affects the epothilone response. Recent evidence suggests that TUBB3 expression is one of the determinants of cell response to paclitaxel (Kavallaris, 2010). Here, we studied if the epothilone drug response changes in cells with modified $\beta$-tubulin isotype levels. We compared the drug responses in TUBB3, TUBB and TUBB2A-C silenced A549 and MCF7 cells and in A549 cells overexpressing Myc-tagged TUBB3 and TUBB to control cells. The cells with manipulated $\beta$-tubulin isotype levels were treated with various concentrations of $\mathrm{mt}$ drugs for $20 \mathrm{~h}$ before analysis of the cellular phenotype. Treatment of TUBB3 silenced A549 cell populations with sagopilone, epothilone $\mathrm{B}$ or ixabepilone induced significantly more multipolar mitotic spindles in comparison to drug-treated control cells transfected with a scrambled siRNA (Figure $3 \mathrm{~B}$ and $\mathrm{D}$ ). The percentage of TUBB3 silenced mitotic cells having multipolar mitotic spindles after $1.0 \mathrm{~nm}$ sagopilone treatment was $77.0 \% \pm 9.5 \%$ compared with $43.0 \% \pm 16.5 \%$ in control cells $(P=0.023)$, after $1.0 \mathrm{~nm}$ epothilone treatment $82.0 \% \pm 9.7 \%$ vs $40.0 \% \pm 10.6 \%$ in controls $(P=0.0002)$ and after $1.0 \mathrm{~nm}$ ixabepilone treatment $39.0 \% \pm 16.2 \%$ vs $3.0 \% \pm 1.0 \%$ in controls $(P=0.025)$, respectively (Figure 3B). The 
A
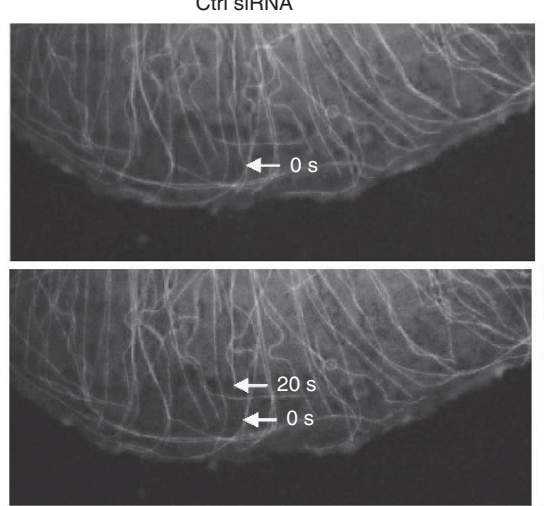

Ctrl siRNA, no drug
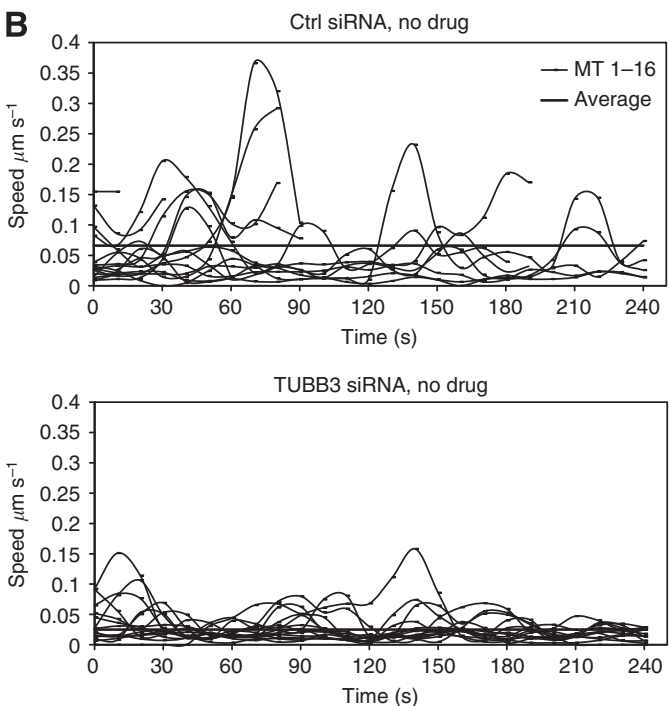

Sagopilone $2 \mathrm{nM}$

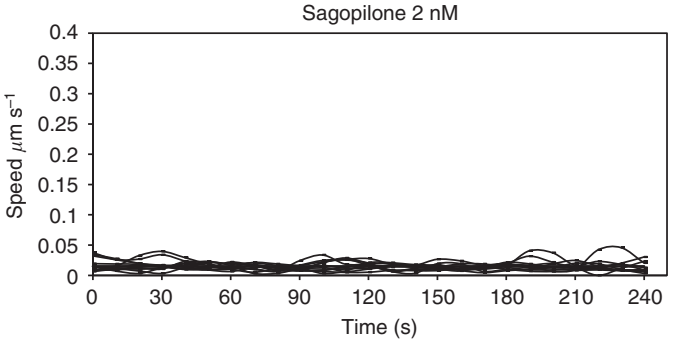

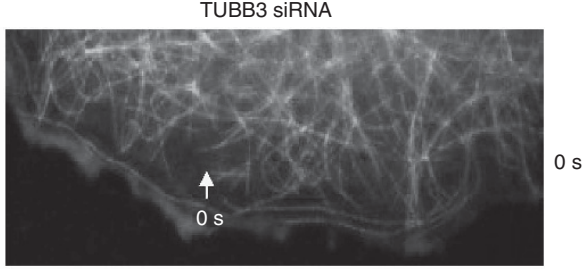

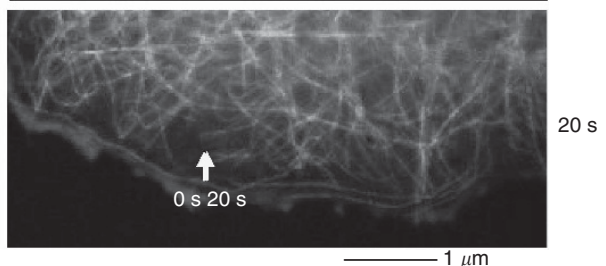

C

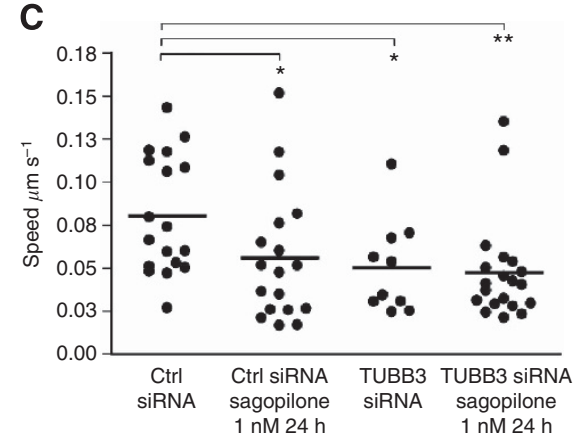

Figure 2. Impact of TUBB3 silencing on mt dynamicity in non-perturbed and sagopilone treated A549 cells. (A) Visualisation of the mt dynamicity (growth and shrinkage rate of $\mathrm{mts}$ ) in live $\alpha$-tubulin-GFP expressing A549 cells $48 \mathrm{~h}$ post-transfection with scrambled non-targeting (Ctrl) or TUBB3 targeting siRNA oligos. The arrows point to the plus end of a selected $\mathrm{mt}$ at $0 \mathrm{~s}$ and $20 \mathrm{~s}$ time points in the control and TUBB3 silenced cell. The scale bar equals $1 \mu \mathrm{m}$. (B) Growth/shrinkage rates of individual mts (speed as $\mu \mathrm{m}$ per second) in one representative cell per each sample. In each cell analysed, the mt dynamics was determined in at least $15 \mathrm{mts}$ (the individual curved lines in each graph). The thick horizontal lines indicate the $\mathrm{mt}$ population dynamicity average. (C) The distribution of the average $\mathrm{mt}$ growth/shrinkage rates (speed as $\mu \mathrm{m}$ per second) from three independent experiments. One dot is the mean value of 6-19 mts analysed per each cell over a period of $240 \mathrm{~s}$ at $10 \mathrm{~s}$ frame capture interval. The bar indicates average $\mathrm{mt}$ dynamicity in the population of analysed cells $\left(n=10-20\right.$ cells). The asterisks point to statistical differences $\left({ }^{\star} P<0.05\right.$, $\star \star P<0.01)$. Only cells in which TUBB3 was effectively depleted $(>95 \%)$ were used in the analysis of mt dynamicity.

result was confirmed using three independent TUBB3 targeting siRNA oligos (Figure 3D). A similar drug sensitisation effect was observed in TUBB3 silenced MCF7 cells exposed to sagopilone $(P=0.0038)$ (Figure 3E). Overexpression of TUBB3-Myc in A549 cells did not rescue the epothilone induced multipolarity (Figure 3C). We conclude that loss of TUBB3 isotype modulates the epothilone drug response by enhancing the sensitivity of cells to the drug effects. In contrast to knockdown of TUBB3, silencing or overexpression of TUBB in A549 or MCF7 cells did not induce significant changes to the spindle morphology in the sagopilone, epothilone B or ixabepilone-treated cells when compared with the drug-treated control cells transfected with a scrambled siRNA (Figure 3B-E). Similarly, loss of TUBB2A-C by RNAi did not significantly alter the mitotic spindle architecture in the presence of the tested $\mathrm{mt}$ drugs in A549 cells ( $P>0.05$, data not shown).

To compare the epothilone results to earlier reports implicating TUBB3 in paclitaxel response (Kavallaris et al, 1997; Stengel et al, 2010), we treated the TUBB3 or TUBB silenced A549 and MCF7 cells with paclitaxel before analysis of the mitotic spindle morphology. Similarly to the cells treated with epothilones, 

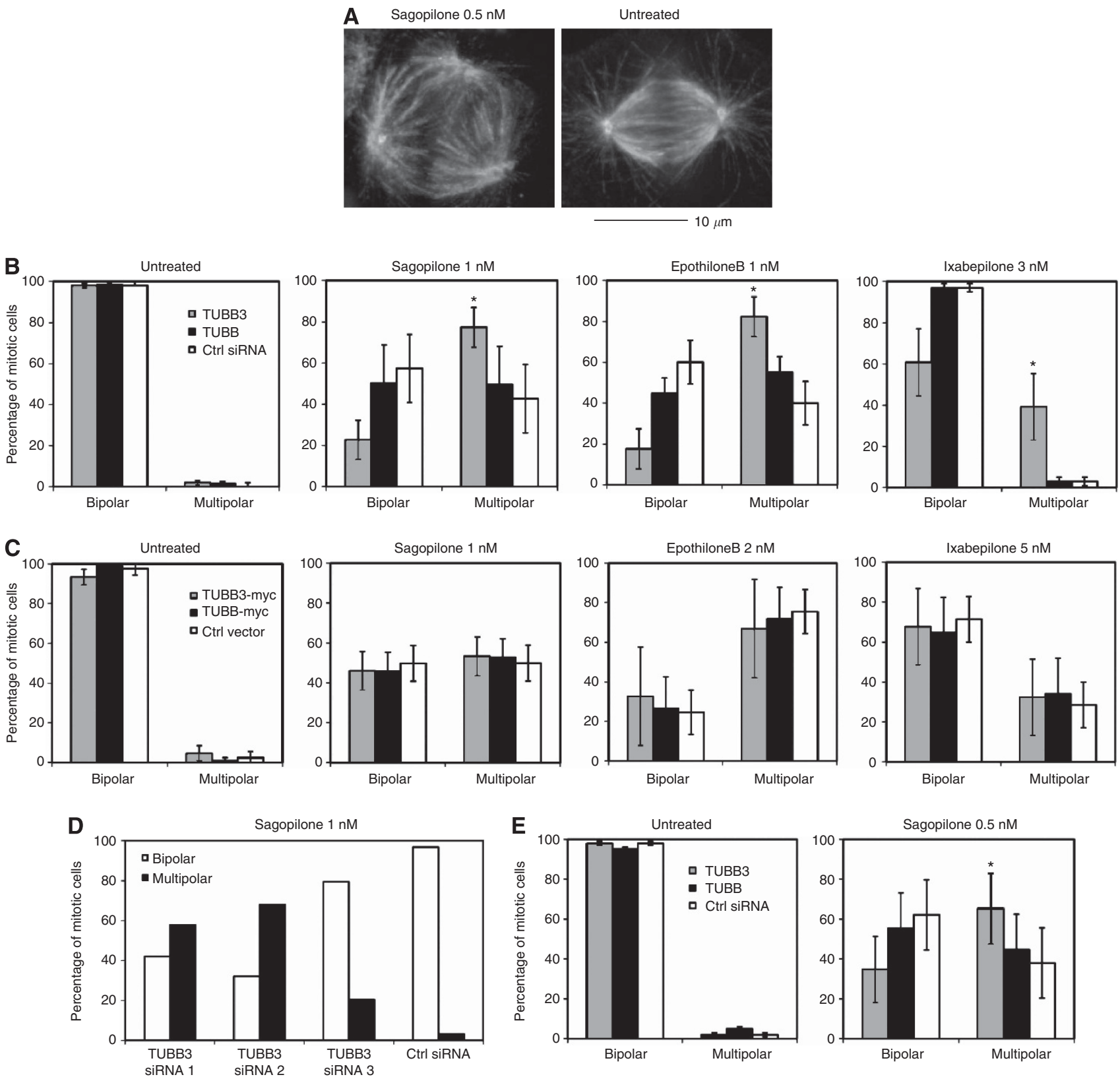

Figure 3. Effects of epothilones on the morphology of the mitotic spindle in TUBB and TUBB3 silenced cells. (A) Multipolarity induced by sagopilone $(0.5 \mathrm{~nm}$ for $20 \mathrm{~h})$ in A549 cells. Green: anti- $\alpha$-tubulin, red: anti-pericentrin, blue: DAPI. Scale bar equals $10 \mu \mathrm{m}$. The diagrams show the effect of depletion (B) or overexpression (C) of TUBB3 and TUBB isotypes on the architecture of mitotic spindles in untreated or drug-treated A549 cells. In each sample at least 150 mitotic cells were categorised into bipolar or multipolar (> 2 poles) classes. Data are from three independent experiments. (D) Silencing of TUBB3 with three different targeting siRNAs increases the multipolarity in the presence of sagopilone in comparison to the control siRNA. (E) The percentage of multipolar mitotic cells in TUBB3 and TUBB silenced MCF7 cells after sagopilone treatment $(0.5 \mathrm{nM}$ $20 \mathrm{~h})$. The asterisks point to statistical differences ( $\left.{ }^{*} P<0.05\right)$.

exposure of TUBB3 silenced cells to $4.0 \mathrm{~nm}$ paclitaxel for $20 \mathrm{~h}$ enhanced the induction of mitotic spindle anomalies compared with control cells. TUBB3 silencing in MCF7 cells elevated the number of cells with multipolar spindles to $22.8 \% \pm 0.2 \%$ from the control level of $9.9 \% \pm 4.1 \%(P=0.044)$ after paclitaxel treatment, while TUBB silenced paclitaxel treated MCF7 cells had only $1.0 \% \pm 1.0 \%$ multipolarity $(P=0.085)$.

TUBB3 removal enhances the inhibition of cell proliferation by epothilones. To analyse if the observed effects of altered $\beta$-tubulin isotype expression on mitotic spindle architecture in the presence of $\mathrm{mt}$ drugs influence the cell proliferation, we determined the $\mathrm{EC}_{50}$ values after various drug treatments in TUBB3 and TUBB silenced cells. Parental A549 cells, mt drug resistant A549EpoB40 cells and MCF7 breast cancer cells were transiently transfected with TUBB3, TUBB or scrambled control siRNA. At $24 \mathrm{~h}$ posttransfection, the cells were exposed to various concentrations of sagopilone, epothilone B or paclitaxel for $48 \mathrm{~h}$. Cell growth was determined using Cell Titer Blue reagent. Loss of TUBB3 enhanced the response of parental A549 cells and MCF7 cells to sagopilone, epothilone B and paclitaxel indicated by the 2-7 fold increase in the $\mathrm{EC}_{50}$ values as compared with the control siRNA (Table 1). TUBB silencing had only a minor sensitising effect in the parental A549 cells to sagopilone and epothilone B (Table 1). In contrast, MCF7 cells were more resistant to epothilones after TUBB removal indicated by the 6 - to 10 -fold increase in the $\mathrm{EC}_{50}$ values (Table 1 ). 
A549EpoB40 cells carry a mutation in the TUBB gene and show resistance against epothilones and paclitaxel (He et al, 2001). TUBB silencing in A549EpoB40 cells resulted in inhibition of cell proliferation at two- to four-fold lower concentrations of sagopilone, epothilone $\mathrm{B}$ and paclitaxel as compared with the controls (Table 1). In contrast, TUBB3 silencing did not alter the cell proliferation response of A549epoB40 cells to the tested drugs (Table 1).

TUBB3 contributes to the epothilone effects on the mitotic spindle. Next, we wanted to clarify the roles of TUBB3 and TUBB as mediators of the drug effect on the mitotic spindle. We studied the effects of both silencing and overexpression of the two tubulin isotypes on the architecture of mitotic spindles in the drug resistant A549EpoB40 cell line. Contrary to the parental A549 cells (Figure 3B), loss of TUBB in A549EpoB40 cells led to an increase of multipolar cells even in the absence of mt drugs while loss of TUBB3 did not cause any notable spindle abnormalities (Figure 4A). We next studied the epothilone effects in the

Table 1. Sagopilone, epothilone $B$ and paclitaxel $E_{50}$ values $(\mathrm{nM})$ in TUBB3 or TUBB silenced A549, A549EpoB40 and MCF7 cells

\begin{tabular}{|l|l|c|c|r|}
\hline Cell line & siRNA & Sagopilone & EpothiloneB & Paclitaxel \\
\hline A549 & CTRL & $1.9 \pm 0.5$ & $2.7 \pm 0.2$ & $5.1 \pm 0.3$ \\
\hline & TUBB3 & $0.6 \pm 0.1$ & $0.6 \pm 0.1$ & $3.0 \pm 0.3$ \\
\hline & TUBB5 & $1.1 \pm 0.1$ & $1.6 \pm 0.2$ & $6.8 \pm 0.7$ \\
\hline A549EpoB40 & CTRL & $10.8 \pm 0.4$ & $20.8 \pm 0.7$ & $44.3 \pm 4.6$ \\
\hline & TUBB3 & $11.5 \pm 0.4$ & $25.0 \pm 0.7$ & $41.2 \pm 2.1$ \\
\hline & TUBB5 & $2.9 \pm 0.3$ & $6.6 \pm 0.3$ & $18.9 \pm 1.4$ \\
\hline MCF7 & CTRL & $0.4 \pm 0.03$ & $0.3 \pm 0.01$ & $4.1 \pm 1.3$ \\
\hline & TUBB3 & $0.1 \pm 0.1$ & $0.1 \pm 0.01$ & $0.6 \pm 0.1$ \\
\hline & TUBB5 & $2.6 \pm 0.3$ & $3.5 \pm 0.8$ & $3.1 \pm 0.3$ \\
\hline Data show mean \pm s.e.m. from six parallel assays. \\
\hline \multicolumn{5}{|l}{}
\end{tabular}
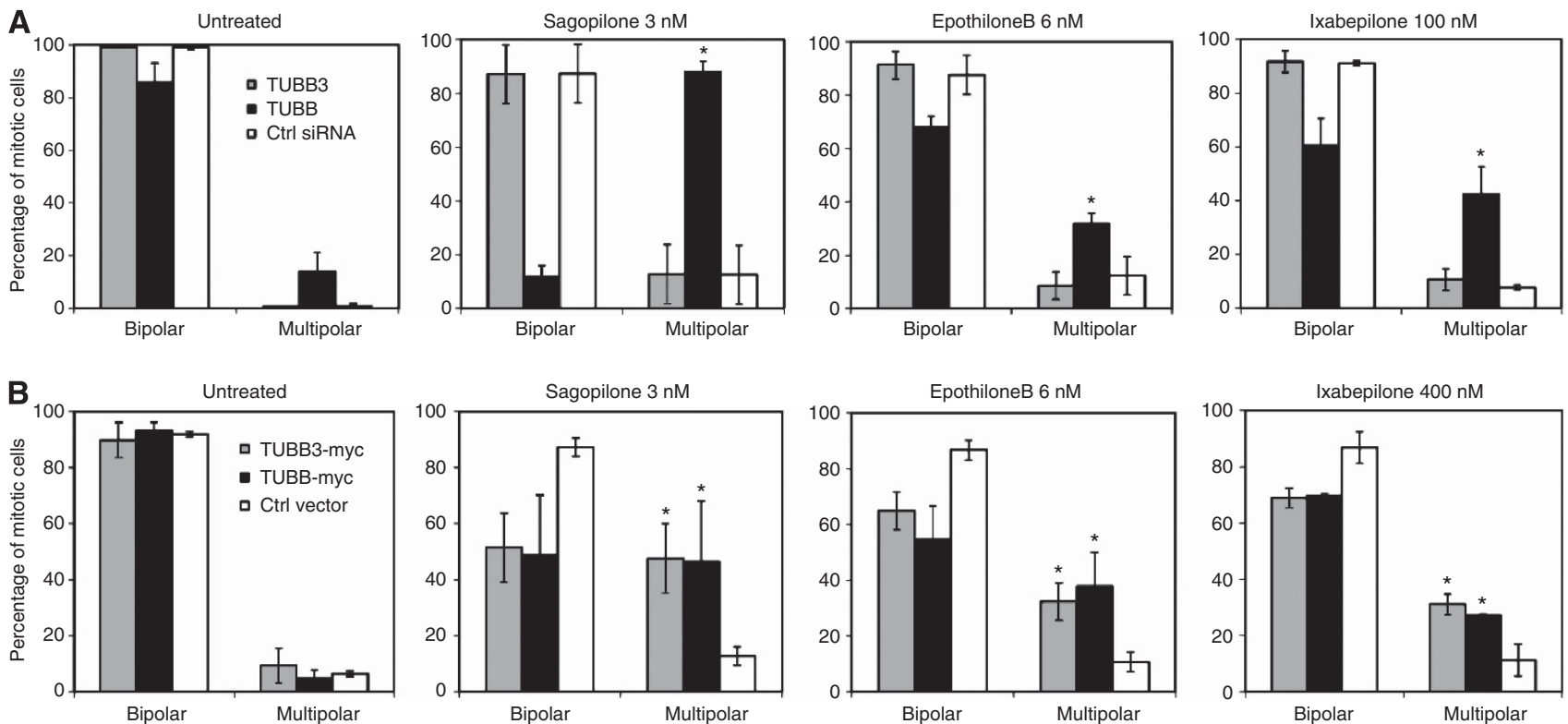

Figure 4. The effects of depletion (A) or overexpression (B) TUBB3 and TUBB isotypes on the architecture of mitotic spindles in the epothilone resistant A549EpoB40 cells after indicated drug treatments At least 150 mitotic cells were analysed per each sample. Data are from three independent experiments. Percentages of mitoses showing either bipolar or multipolar ( $>2$ poles) mitotic spindles are shown. The asterisks point to statistical differences ( ${ }^{\star} P<0.05$ ). 
We analysed the mitotic spindle architecture in epothilone treated and tubulin isotype silenced cells in order to better understand the drug actions in relation to the cells' $\mathrm{mt}$ constituents and the cellular mechanisms inhibiting cell proliferation. Earlier studies have indicated that low taxol concentrations disrupt the normal mt cytoskeleton and cell cycle without affecting the total tubulin mass (Torres and Horwitz, 1998). Moreover, the penetrance of $\mathrm{mt}$ drugs inside the tumour in vivo may often be suboptimal lowering the intracellular drug concentrations after chemotherapy. For these reasons we used in our study the lowest drug concentrations that produced a clear mitotic response. Importantly, the low epothilone concentrations we used in the study did not induce dramatic cell-cycle effects but were sufficient to cause anomalies in the mt dynamics resulting in multipolar mitosis, perturbation of cytokinesis and multinucleation, which all are defects that likely contribute to the drug-induced cell death.

The increased sensitivity of TUBB3 silenced cells to mt binding drugs could be partly explained by the observed decrease in the $\mathrm{mt}$ dynamics. Suppressed mt growth or shrinkage rates in TUBB3 silenced cells did not cause major mitotic defects, but are probably sufficient to enhance the effects of epothilones on the overall $\mathrm{mt}$ dynamics. In a recent publication by Gan et al (2011), the authors did not observe significant changes in the $\mathrm{mt}$ dynamic instability caused by lack of TUBB3 in non-small cell lung cancer cells. In our analysis we detected considerable variation in the $\mathrm{mt}$ dynamics between individual cells in the TUBB3 siRNA treated population. We believe that a technical difference in the analysis of the $\mathrm{mt}$ dynamics between the two studies provides an explanation to the discrepancy in the results. To avoid potential phenotype masking effects caused by partially silenced cells, we confirmed in our study by anti-TUBB3 immunofluorescence that the intensity of TUBB3 staining was below the level of detection in each cell we used for the determination of $\mathrm{mt}$ dynamics in the TUBB3 silenced cell population. In other words, our study focused only to cells in which the silencing efficacy was the highest possible. It is also possible that the different cell models used in the two studies have an impact on the results.

Our data show that the exogenously expressed Myc-tagged TUBB3 and TUBB incorporate into the mt filaments, but they cause only minor effects on the drug response in the A549 cells. Due to the endogenous expression of tubulin isotypes it is possible that the change in the isotype composition in the mt lattice as a consequence of exogenous expression of TUBB3 or TUBB is not as dramatic as the depletion of individual tubulin isotypes. In the A549 and MCF7 cells, the endogenous expression levels of the TUBB3 and TUBB are intermediate in the affymetrix data set from 81 human cell lines analysed (unpublished data). In previous publications there has been increased paclitaxel resistance following exogenous human TUBB3 in Chinese hamster ovarian cells, but these cells do not express endogenous TUBB3 at all (Hari et al, 2003). Also, we cannot exclude the possibility that additional mechanisms would exist to control the tubulin isotype constitutes in the various types of $\mathrm{mts}$ of interphase and mitotic cells. However, the overexpression of TUBB3 in the TUBB mutated A549EpoB40 cell line slightly reversed the epothilone resistant phenotype, suggesting that the increased TUBB3 proportion in the mt filaments can functionally compensate the mutated TUBB. Indirectly this proposes that epothilones can interact with the TUBB3 isotype and mediate mt stabilisation through a TUBB3- $\alpha$ tubulin dimer.

Epothilone B and its synthetic derivatives ixabepilone and sagopilone are reported to bind in the paclitaxel binding site of $\beta$-tubulins but the exact interaction footprint of these drugs is slightly different (Giannakakou et al, 2000). The binding mechanisms and affinities vary also between epothilones (KhrapunovichBaine et al, 2011). Thus, we investigated if the cellular effects caused by altered $\beta$-tubulin isotype expression are similar between epothilone B, sagopilone, ixabepilone and earlier reported paclitaxel responses. For the first time, a significant correlation was found between TUBB3 levels and drug response with all the tested epothilones. A recent publication reported increased cell death in TUBB3 silenced cells after epothilone B treatment which is in line with our results (Gan et al, 2011). Additionally, a HeLa cell line constitutively overexpressing TUBB3 has been shown to be less sensitive to epothilone B (Risinger et al, 2008). On the other hand, it has been shown that TUBB3 levels do not increase after Epothilone B treatment, which has been reported to occur after paclitaxel treatment (Mozzetti et al, 2008). In another report, the authors found that uterine and ovarian cell lines are equally responsive to paclitaxel, but uterine cell lines were more sensitive to epothilone B. Also, they found higher TUBB3 expression in uterine $v s$ ovarian samples (Carrara et al, 2012). The controversy between different studies underlines the need for further studies to reveal the detailed mechanisms of TUBB3 effects.

Analysis of the epothilone binding sites in human $\beta$-tubulin isotypes has indicated that differences exists in the residues constituting the epothilone binding footprint between TUBB3 and other $\beta$-tubulin isotypes (Huzil et al, 2006; Magnani et al, 2006). This variation in the drug binging can affect the $m t$ stabilisation by epothilones. However, TUBB3 silencing is also shown to increase cell death in response to vincristine, which binds $\beta$-tubulin closer to the inter-dimer interphase of mts (Huzil et al, 2006). We speculate that the impact of TUBB3 on the epothilone response is mainly mediated through suppressed $\mathrm{mt}$ dynamics and/or conformational changes in the mt filaments rather than via differences in the direct drug binding affinity between the epothilones and $\beta$-tubulin isotypes. Interestingly, TUBB3 expression has been reported to also affect doxorubicin and cisplatin responses, which suggests that additional indirect mechanisms may contribute to the role of TUBB3 in drug response (Gan et al, 2007; Galmarini et al, 2008). For example, very recently two GTPases were reported to interact with TUBB3, one of which is implicated in the regulation of the prosurvival factor PIM1 (De Donato et al, 2012).

Sagopilone is in active development phase and ixapebilone is accepted for clinical use in the treatment of breast cancer, and is also being clinically evaluated in the treatment of non-small cell lung cancer and prostate cancer (Liu et al, 2012; Smith, 2012). The $\beta$-tubulin isotype composition that varies in $\mathrm{mt}$ lattice between cells, tissues and individuals (Seve et al, 2005; Kilpinen et al, 2008) is most likely an important post-translational control mechanism of mt function and now shown here as one determinant of the epothilone response. Our results demonstrating the effects of altered $\beta$-tubulin isotype expression on the cells' $\mathrm{mt}$ dynamics and epothilone response have implications for design of targeted therapies where the $\beta$-tubulin expression spectrum of individual patient malignancies can reflect the tumours cells' response to $\mathrm{mt}$ binding drugs. In future, analysis of the patients' tumour cells' $\beta$-tubulin isotype expression pattern may assist in making personalised therapy decisions in certain cancer types.

\section{ACKNOWLEDGEMENTS}

This study was supported by the Academy of Finland (Grant 131946), The Finnish Cancer Organisations, and the Cancer Foundation of Southwest Finland. Pauliina Toivonen and Agnieszka Grzegorzewska are acknowledged for technical assistance. We thank Bayer HealthCare for providing the sagopilone.

\section{REFERENCES}

Alvarez M, Paull K, Monks A, Hose C, Lee JS, Weinstein J, Grever M, Bates S, Fojo T (1995) Generation of a drug resistance profile by quantitation of 
mdr-1/P-glycoprotein in the cell lines of the National Cancer Institute Anticancer Drug Screen. J Clin Invest 95: 2205-2214.

Berrieman HK, Lind MJ, Cawkwell L (2004) Do beta-tubulin mutations have a role in resistance to chemotherapy? Lancet Oncol 5: 158-164.

Burkhart CA, Kavallaris M, Band Horwitz S (2001) The role of beta-tubulin isotypes in resistance to antimitotic drugs. Biochim Biophys Acta 1471: O1-O9.

Carrara L, Guzzo F, Roque DM, Bellone S, Emiliano C, Sartori E, Pecorelli S, Schwartz PE, Rutherford TJ, Santin AD (2012) Differential in vitro sensitivity to patupilone versus paclitaxel in uterine and ovarian carcinosarcoma cell lines is linked to tubulin-beta-III expression. Gynecol Oncol 125: 231-236.

De Donato M, Mariani M, Petrella L, Martinelli E, Zannoni GF, Vellone V, Ferrandina G, Shahabi S, Scambia G, Ferlini C (2012) Class III betatubulin and the cytoskeletal gateway for drug resistance in ovarian cancer. J Cell Physiol 227: 1034-1041.

Downing KH (2000) Structural basis for the interaction of tubulin with proteins and drugs that affect microtubule dynamics. Annu Rev Cell Dev Biol 16: 89-111.

Galmarini CM, Treilleux I, Cardoso F, Bernard-Marty C, Durbecq V, Gancberg D, Bissery MC, Paesmans M, Larsimont D, Piccart MJ, Di Leo A, Dumontet C (2008) Class III beta-tubulin isotype predicts response in advanced breast cancer patients randomly treated either with single-agent doxorubicin or docetaxel. Clin Cancer Res 14: 4511-4516.

Gan PP, McCarroll JA, Byrne FL, Garner J, Kavallaris M (2011) Specific beta-tubulin isotypes can functionally enhance or diminish epothilone B sensitivity in non-small cell lung cancer cells. PLoS One 6: e21717.

Gan PP, Pasquier E, Kavallaris M (2007) Class III beta-tubulin mediates sensitivity to chemotherapeutic drugs in non small cell lung cancer. Cancer Res 67: 9356-9363.

Giannakakou P, Gussio R, Nogales E, Downing KH, Zaharevitz D, Bollbuck B, Poy G, Sackett D, Nicolaou KC, Fojo T (2000) A common pharmacophore for epothilone and taxanes: molecular basis for drug resistance conferred by tubulin mutations in human cancer cells. Proc Natl Acad Sci USA 97: 2904-2909.

Giannakakou P, Sackett DL, Kang YK, Zhan Z, Buters JT, Fojo T, Poruchynsky MS (1997) Paclitaxel-resistant human ovarian cancer cells have mutant beta-tubulins that exhibit impaired paclitaxel-driven polymerization. J Biol Chem 272: 17118-17125.

Hari M, Yang H, Zeng C, Canizales M, Cabral F (2003) Expression of class III beta-tubulin reduces microtubule assembly and confers resistance to paclitaxel. Cell Motil Cytoskeleton 56: 45-56.

He L, Yang CP, Horwitz SB (2001) Mutations in beta-tubulin map to domains involved in regulation of microtubule stability in epothilone-resistant cell lines. Mol Cancer Ther 1: 3-10.

Huzil JT, Ludueña RF, Tuszynski J (2006) Comparative modelling of human $\beta$ tubulin isotypes and implications for drug binding. Nanotechnology 17: 90-90-100.

Jordan MA, Wilson L (2004) Microtubules as a target for anticancer drugs. Nat Rev Cancer 4: 253-265.

Kanda T, Sullivan KF, Wahl GM (1998) Histone-GFP fusion protein enables sensitive analysis of chromosome dynamics in living mammalian cells. Curr Biol 8: 377-385.

Kavallaris M (2010) Microtubules and resistance to tubulin-binding agents. Nat Rev Cancer 10: 194-204.

Kavallaris M, Kuo DY, Burkhart CA, Regl DL, Norris MD, Haber M, Horwitz SB (1997) Taxol-resistant epithelial ovarian tumors are associated with altered expression of specific beta-tubulin isotypes. J Clin Invest 100: 1282-1293.
Khrapunovich-Baine M, Menon V, Yang CP, Northcote PT, Miller JH, Angeletti RH, Fiser A, Horwitz SB, Xiao H (2011) Hallmarks of molecular action of microtubule stabilizing agents: effects of epothilone $\mathrm{B}$, ixabepilone, peloruside $\mathrm{A}$, and laulimalide on microtubule conformation. J Biol Chem 286: 11765-11778.

Kilpinen S, Autio R, Ojala K, Iljin K, Bucher E, Sara H, Pisto T, Saarela M, Skotheim RI, Bjorkman M, Mpindi JP, Haapa-Paananen S, Vainio P, Edgren H, Wolf M, Astola J, Nees M, Hautaniemi S, Kallioniemi O (2008) Systematic bioinformatic analysis of expression levels of 17,330 human genes across 9,783 samples from 175 types of healthy and pathological tissues. Genome Biol 9: R139.

Liu G, Chen YH, Dipaola R, Carducci M, Wilding G (2012) Phase II Trial of weekly ixabepilone in men with metastatic castrate-resistant prostate cancer (E3803): A Trial of the Eastern Cooperative Oncology Group. Clin Genitourin Cancer 10(2): 99-105.

Magnani M, Ortuso F, Soro S, Alcaro S, Tramontano A, Botta M (2006) The betaI/betaIII-tubulin isoforms and their complexes with antimitotic agents. Docking and molecular dynamics studies. FEBS J 273: 3301-3310.

Mozzetti S, Iantomasi R, De Maria I, Prislei S, Mariani M, Camperchioli A, Bartollino S, Gallo D, Scambia G, Ferlini C (2008) Molecular mechanisms of patupilone resistance. Cancer Res 68: 10197-10204.

Ranganathan S, Benetatos CA, Colarusso PJ, Dexter DW, Hudes GR (1998) Altered beta-tubulin isotype expression in paclitaxel-resistant human prostate carcinoma cells. Br J Cancer 77: 562-566.

Risinger AL, Jackson EM, Polin LA, Helms GL, LeBoeuf DA, Joe PA, HopperBorge E, Luduena RF, Kruh GD, Mooberry SL (2008) The taccalonolides: microtubule stabilizers that circumvent clinically relevant taxane resistance mechanisms. Cancer Res 68: 8881-8888.

Seve P, Mackey J, Isaac S, Tredan O, Souquet PJ, Perol M, Lai R, Voloch A, Dumontet C (2005) Class III beta-tubulin expression in tumor cells predicts response and outcome in patients with non-small cell lung cancer receiving paclitaxel. Mol Cancer Ther 4: 2001-2007.

Smith NZ (2012) Treating metastatic breast cancer with systemic chemotherapies. Clin J Oncol Nurs 16: E33-E43.

Stengel C, Newman SP, Leese MP, Potter BV, Reed MJ, Purohit A (2010) Class III beta-tubulin expression and in vitro resistance to microtubule targeting agents. Br J Cancer 102: 316-324.

Tommasi S, Mangia A, Lacalamita R, Bellizzi A, Fedele V, Chiriatti A, Thomssen C, Kendzierski N, Latorre A, Lorusso V, Schittulli F, Zito F, Kavallaris M, Paradiso A (2007) Cytoskeleton and paclitaxel sensitivity in breast cancer: the role of beta-tubulins. Int J Cancer 120: 2078-2085.

Torres K, Horwitz SB (1998) Mechanisms of Taxol-induced cell death are concentration dependent. Cancer Res 58: 3620-3626.

Wartmann M, Altmann KH (2002) The biology and medicinal chemistry of epothilones. Curr Med Chem Anticancer Agents 2: 123-148.

Wiesen KM, Xia S, Yang CP, Horwitz SB (2007) Wild-type class I beta-tubulin sensitizes Taxol-resistant breast adenocarcinoma cells harboring a betatubulin mutation. Cancer Lett 257: 227-235.

Yang CP, Verdier-Pinard P, Wang F, Lippaine-Horvath E, He L, Li D, Hofle G, Ojima I, Orr GA, Horwitz SB (2005) A highly epothilone B-resistant A549 cell line with mutations in tubulin that confer drug dependence. Mol Cancer Ther 4: 987-995.

This work is published under the standard license to publish agreement. After 12 months the work will become freely available and the license terms will switch to a Creative Commons AttributionNonCommercial-Share Alike 3.0 Unported License.

Supplementary Information accompanies this paper on British Journal of Cancer website (http://www.nature.com/bjc) 